Rev. Biol. Neotrop. 8(2): 23-31. 2011

\title{
itossociologia de Macrófitas Aquáticas associadas ao Rio Miranda, Pantanal, MS, Brasil
}

\section{Carlos Rodrigo Lehn}

Instituto Federal de Educação, Ciência e Tecnologia de Mato Grosso do Sul - IFMS, Campus Coxim, Rua Pereira Gomes 355, 79400-000, Bairro Novo Mato Grosso, Coxim, Mato Grosso do Sul, Brasil; e-mail: crlehn@gmail.com

\section{Marcelo Leandro Bueno}

Universidade Federal de Minas Gerais, Departamento de Botânica, Avenida Antônio Carlos 6627, Cx. Postal 486, 31270-901, Pampulha, Belo Horizonte, Minas Gerais, Brasil.

\section{Deborah Christiane Leite Kufner}

Universidade Federal de Minas Gerais - UFMG, Departamento de Botânica, Avenida Antônio Carlos 6627, Cx. Postal 486, 31270-901, Pampulha, Belo Horizonte, Minas Gerais, Brasil.

\section{Edna SCRemin-Dias}

Universidade Federal de Mato Grosso do Sul - UFMS, Departamento de Biologia, Avenida Senador Filinto Müller s/n, Cidade Universitária, 79070-900, Campo Grande, Mato Grosso do Sul, Brasil.

\author{
Vali Joana Pott \\ Universidade Federal de Mato Grosso do Sul - UFMS, Departamento de Biologia, Avenida \\ Senador Filinto Müller s/n, Cidade Universitária, 79070-900, Campo Grande, Mato Grosso \\ do Sul, Brasil.

\section{Geraldo Alves Damasceno Junior} \\ Universidade Federal de Mato Grosso do Sul - UFMS, Departamento de Biologia, Avenida \\ Senador Filinto Müller s/n, Cidade Universitária, 79070-900, Campo Grande, Mato Grosso \\ do Sul, Brasil.
}

\begin{abstract}
Resumo: Analisou-se quantitativamente uma comunidade de macrófitas aquáticas associada ao Rio Miranda, Pantanal, Mato Grosso do Sul utilizando dois métodos de amostragem: interseção linear e parcelas. Ao todo foram catalogadas 36 espécies, distribuídas em 31 gêneros e 20 famílias, sendo Poaceae a família com maior riqueza específica (5 spp.), seguida por Malvaceae (4), Asteraceae, Araceae, Convolvulaceae e Polygonaceae representadas por três espécies cada. Polygonum acuminatum foi a espécie que apresentou maior valor de importância (IVI) para ambos os métodos. O índice de diversidade de Shannon $\left(H^{\prime}\right)$ foi de 2,44 para o método da interseção linear e de 2,47 para o método das parcelas. Para ambos os métodos, a curva de rarefação demonstrou tendência à estabilização, indicando suficiência amostral. Os resultados obtidos foram similares para as duas metodologias, indicando ambas são apropriadas para estudos quantitativos com esta comunidade.
\end{abstract}

Palavras-chave: Planta aquática, área úmida, ecologia vegetal, riqueza de espécies.

Aвstract: Phytosociology of aquatic macrophytes associated to the Miranda river, Pantanal, MS, Brazil. - This study aimed to analyze quantitatively the community structure of aquatic macrophytes associated to the Miranda river, Pantanal wetland, Mato Grosso do Sul, utilizing two sampling methods: line interception and plots. A total of 36 species were recorded, distributed in 31 genera and 20 families, Poaceae being the family with highest species richness ( 5 spp.), followed by Malvaceae (4), Asteraceae, Araceae, Convolvulaceae e Polygonaceae represented by three species each one. Polygonum acuminatum showed highest importance value under both techniques. Shannon diversity index $\left(\mathrm{H}^{\prime}\right)$ was 2.44 for line interception and 2.47 for plots. The rarefaction curve showed stabilization trend for both methods, indicating sampling sufficiency. The results were similar for both techniques, suggesting that either is appropriate for quantitative surveys of this vegetation.

KEYwords: Aquatic plant, floodplain, plant ecology, species richness. 


\section{INTRODUÇÃO}

O Pantanal, planície alagável com cerca de 140.000 km², apresenta vegetação descontínua (em manchas) entremeada com corpos d'água (Salis, 2000). A maior parte dos ambientes alagáveis do Pantanal compreende áreas de campo, bastando alguns dias ou semanas de inundação para que as plantas aquáticas apareçam (Hamilton et al., 1996). Segundo Pedralli (1992), a região pantaneira é indicada como um dos quatro centros de diversidade de macrófitas aquáticas do Brasil.

As macrófitas desempenham um importante papel nos ecossistemas aquáticos, cumprindo, juntamente com as microalgas, a função de produtoras primárias, participando da ciclagem e estocagem de nutrientes, da formação de detritos orgânicos e do controle da poluição e da eutrofização artificial (Pott \& Pott, 2000). Além disso, também promovem a diversificação de habitats, criando locais de abrigo e alimentação para fauna e substrato natural para a formação de perifíton (Esteves, 1998). Em rios e riachos, as macrófitas influenciam na sedimentação e retenção de nutrientes, nas características físicas e químicas da água, assim como, em alguns casos, afetam significativamente a velocidade de fluxo da água (Schulz et al., 2003).

O número de estudos envolvendo macrófitas aquáticas no Brasil é crescente, especialmente levando-se em consideração as duas últimas décadas, onde os principais motivos que contribuíram para esse cenário foram o interesse pela biodiversidade e a necessidade de manejo dos ecossistemas aquáticos (Thomaz \& Bini, 2003).

A riqueza de macrófitas aquáticas no Pantanal é estimada em mais de 240 espécies (Pott $\&$ Pott, 2000). Embora seja considerada uma das áreas úmidas mais importantes do planeta, as macrófitas aquáticas são ainda pouco estudadas quantitativamente na região, salvo os trabalhos realizados por Pott et al. (1989, 1992, 1999), Prado et al. (1992), Frey (1995) e Schessl (1999).

O presente estudo tem como objetivo amostrar quantitativamente as macrófitas aquáticas ocorrentes em uma área situada às margens do Rio Miranda, no Pantanal Sul-matogrossense, município de Corumbá, MS, visando contribuir para o conhecimento deste grupo na área de estudo.
Material e métodos

\section{Caracterização da Área de estudo}

Localiza-se às margens do Rio Miranda, entre as coordenadas $19^{\circ} 34^{\prime} 30.20^{\prime \prime} \mathrm{S} /$ $57^{\prime} 0^{\prime} 16.82^{\prime \prime} \mathrm{W}$ e $19^{\circ} 34^{\prime} 34.07^{\prime \prime} \mathrm{S} / 57^{\prime} 0^{\prime} 18.74^{\prime \prime} \mathrm{W}$ distando a área de estudo cerca de $3 \mathrm{~km}$ à montante da Base de Estudos do Pantanal $\mathrm{BEP}$, pertencente à Universidade Federal de Mato Grosso do Sul - UFMS e situada na região conhecida como Passo do Lontra, Pantanal do Miranda, município de Corumbá, MS (Figura 1).

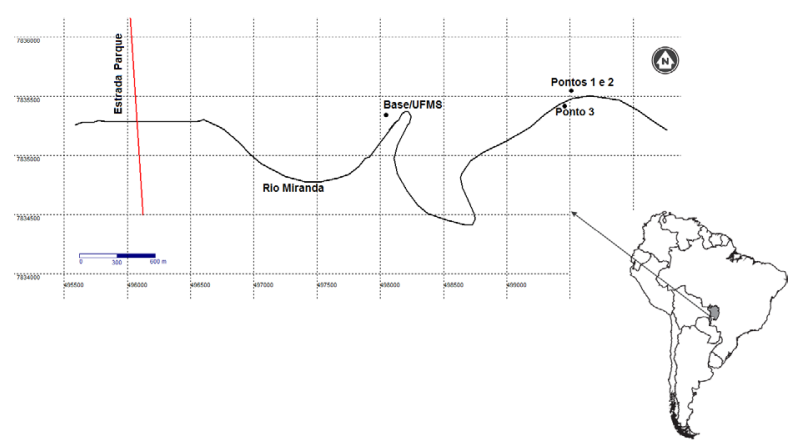

Figura 1: Localização da área de estudo nas proximidades da Base de Estudos da Universidade Federal de Mato Grosso do Sul, Passo do Lontra, Pantanal, MS, Brasil.

Segundo a classificação climática de Köppen (1948), o clima da região é do tipo $A w$, megatérmico, com inverno seco e verão chuvoso. As médias anuais de precipitação e temperatura, no período 1975-1996 foram 1077 mm e $25,1^{\circ} \mathrm{C}$, respectivamente. A maior precipitação média ocorre em janeiro, com $191 \mathrm{~mm}$ e a menor em julho com $19 \mathrm{~mm}$, apresentando uma deficiência anual hídrica de $318 \mathrm{~mm}$. A temperatura máxima absoluta atinge $40^{\circ} \mathrm{C}$ nos meses de outubro a janeiro, e as mínimas absolutas podem se aproximar a $0{ }^{\circ} \mathrm{C}$ nos meses de maio a agosto (Soriano, 1999).

\section{Amostragem fitossociológica}

Os principais métodos empregados para estudos quantitativos com o estrato herbáceo são da parcela, linha ou ponto. Dentre estes estão o da interseção linear, que foi originalmente descrito por Tansley \& Chipp (1926) e o método das parcelas (Mueller-Dombois \& Ellenberg, 1974). Estes métodos têm sido amplamente utilizados em levantamentos do estrato 
herbáceo de ecossistemas brasileiros (Munhoz \& Felfili 2004, 2006a, 2006b, 2007, 2008) e não-brasileiros (Andrianarivo, 1993; Nemati \& Goetz, 1995; Wolf, 1998).

Para realização do presente estudo foram utilizados dois métodos, sendo estes o método da interseção linear e método das parcelas. $\mathrm{O}$ método da interseção linear consiste em traçar linhas sobre a vegetação a ser amostrada e anotar o comprimento em que a linha é interceptada por uma espécie, que dividido pelo comprimento total das espécies inventariadas sob as linhas, estima a proporção da área coberta por aquela espécie (Munhoz \& Felfili, 2006a).

Neste estudo foram estabelecidas $30 \mathrm{li}$ nhas, distribuídas em três pontos amostrais, os quais estão sujeitos a inundação periódica pelo Rio Miranda. As linhas apresentavam $5 \mathrm{~m}$ de comprimento, foram dispostas paralelas entre si e em relação ao leito do rio (Figura 2), sendo que cada segmento de 1 metro corresponde a uma unidade amostral (UA) para a análise fitossociológica, totalizando 150 unidades amostrais. A amostragem através do método das parcelas se deu utilizando um quadrado de 1 $\mathrm{m}^{2}$ de área confeccionado com canos de PVC, onde cada quadrado constitui uma unidade. As parcelas foram implantadas contiguamente nos mesmos pontos amostrados com o método da interseção linear. A cobertura de cada espécie nas parcelas foi obtida através de estimativa visual do percentual de cobertura, em um total de 85 parcelas amostradas. Ponto 1
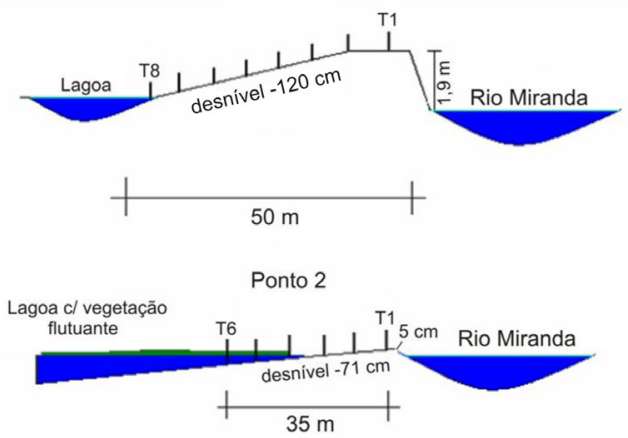

Ponto 3

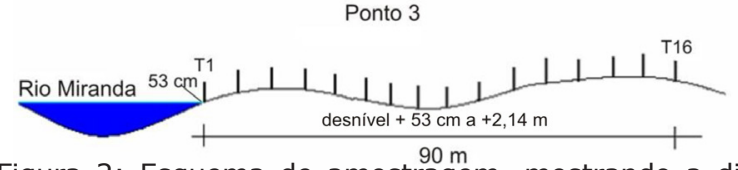

Figura 2: Esquema de amostragem, mostrando a distância dos transectos implantados em relação ao leito do Rio Miranda nos três pontos de amostragem, para a aplicação da metodologia de Parcela e Interseção linear, Pantanal, MS, Brasil.
Os parâmetros fitossociológicos (coberturas absoluta e relativa, freqüências absoluta e relativa e índice sintético do valor de importância) foram estabelecidos de acordo com Meirelles et al. (2002)e Damasceno-Junior \& Pott (2011) e calculados utilizando-se a planilha Excel Microsoft versão 2007.

A cobertura absoluta (CA) de cada espécie foi determinada a partir da seguinte fórmula:

$\mathrm{CA}=\left(1_{\mathrm{i}} / \mathrm{L}\right) \times 100$, onde:

$1_{i}=$ comprimento interceptado pela espécie $\mathrm{i}(\mathrm{cm})$ em todas as unidades amostrais. No caso das parcelas percentual que a espécie ocupa em cada unidade amostral;

$\mathrm{L}=$ comprimento total da linha. Para as parcelas área total amostrada;

A cobertura linear relativa (CR) foi determinada a partir da seguinte fórmula:

$\mathrm{CR}=\left(\mathrm{CA}_{\mathrm{i}} / \sum \mathrm{CA}\right) \times 100$, onde:

$\mathrm{CA}_{\mathrm{i}}=$ cobertura absoluta da espécie $\mathrm{i}$;

$\Sigma \mathrm{CA}=$ somatório das coberturas absolutas de todas as espécies amostradas na área de estudo.

A freqüência absoluta (FA) foi determinada a partir da seguinte fórmula:

$\mathrm{FA}_{\mathrm{i}}=\left(\mathrm{J}_{\mathrm{i}} / \mathrm{N}\right) \times 100$, onde:

$\mathrm{J}_{1}=$ número de unidades amostrais e/ou parcelas que a espécie i aparece.

$\mathrm{N}=$ número total de unidades amostrais.

A freqüência relativa (FR) foi determinada a partir da seguinte fórmula:

$\mathrm{FR}_{\mathrm{i}}=\left(\mathrm{FA}_{\mathrm{i}} / \sum \mathrm{FA}\right) \times 100$, onde $\sum \mathrm{FA}$ representa o somatório de todas as frequências absolutas das espécies amostradas na área de estudo.

Para avaliar a diversidade florística em cada ponto amostrado foi realizada uma adaptação do índice de diversidade de Shannon $\left(\mathrm{H}^{\prime}\right)$, utilizando-se ao invés do número de indivíduos os valores de cobertura (CA) observados durante a amostragem, conforme adotado por Munhoz \& Felfili (2006). O índice de Shannon considera a abundância proporcional de espécies em uma amostra e para o seu cálculo em geral utiliza-se a proporção de indivíduos por espécie (Munhoz \& Felfili, 2006a). A abundância pode ser avaliada pelo 
número de indivíduos e por outras medidas de ocupação do espaço pelas plantas, como a freqüência e a cobertura (Munhoz \& Felfili 2006a). Neste caso, optou-se pelo uso da proporção de cobertura de espécie como uma medida de abundância, pois para o estrato herbáceo-subarbustivo a definição de indivíduo é difícil, uma vez que rebrotos basais (rametes) de um mesmo indivíduo são comuns.

$$
\mathrm{H}^{\prime}=\sum(\mathrm{Pi} .(\mathrm{LnPi}))
$$

onde: Pi é a proporção de cobertura de cada espécie $(\mathrm{Pi}=\mathrm{CAi} / \mathrm{N}) ; \mathrm{CA}=$ cobertura linear absoluta da espécie $i ; \mathrm{N}=$ soma da cobertura de todas as espécies encontradas na área e $\mathrm{Ln}=$ logaritmo neperiano.

Com base nos valores de freqüência absoluta, foi estabelecida uma curva de rarefação, buscando verificar a suficiência amostral. A curva de rarefação foi calculada utilizando-se o software PAST 2.03 (Hammer et al., 2001).

Identificação - As espécies foram identificadas utilizando-se bibliografia especializada, através da comparação com exsicatas depositadas no herbário CGMS ou consulta à especialistas. Os nomes dos autores das espécies foram confirmados utilizando-se o banco de dados do $\mathrm{W}^{3}$ Tropicos do Jardim Botânico do Missouri/EUA (www.tropicos.org). As formas biológicas das espécies seguiram o adotado por Pott \& Pott (2000).

\section{Resultados e discussão}

Ao todo foram catalogadas 36 espécies, distribuídas em 31 gêneros e 20 famílias. As famílias que apresentaram maior riqueza de espécies foram, respectivamente, Poaceae (5 espécies), Malvaceae (4), Asteraceae, Araceae, Convolvulaceae e Polygonaceae representadas por três espécies cada. A exceção das famílias Cucurbitaceae e Salviniaceae representadas por duas espécies cada, as demais famílias estavam representadas por apenas uma espécie.

Assim como observado neste trabalho, outros estudos também apontam para a importância florística da família Poaceae em comunidades hidrófitas, tanto no Pantanal (Junk \& Piedade, 1993; Pott \& Pott, 2000; Pivari et al., 2008), quanto em outras áreas úmidas do Brasil (Irgang et al., 1984; Bove et al., 2003; Meirelles et al., 2004).

Polygonum acuminatum, Commelina schomburgkiana, Merremia umbellata e Panicum elephantipes foram, respectivamente, as espécies que mais se destacaram na comunidade, considerando o valor de importância, para ambos os métodos, representando cerca de $50 \%$ do VI total (Tabelas 1 e 2 ). 
Tabela 1: Parâmetros fitossociológicos (Coberturas absoluta (CA) e relativa (CR), freqüências absoluta (FA) e relativa (FR) e valor de importância (VI)) obtidos através do método da interseção linear para a comunidade de macrófitas aquáticas amostrada às margens do rio Miranda, Pantanal, MS, Brasil.

\begin{tabular}{|c|c|c|c|c|c|c|}
\hline Espécie & Família & CA & CR & FA & $\mathbf{F R}$ & VI \\
\hline Polygonum acuminatum Kunth & Polygonaceae & 1444,7 & 23,17 & 64,2 & 10,27 & 33,45 \\
\hline Commelina schomburgkiana Klotzsch & Commelinaceae & 1143,02 & 18,33 & 45,02 & 7,22 & 25,56 \\
\hline Merremia umbellata (L.) Hallier f. & Convolvulaceae & 904,15 & 14,50 & 52,1 & 8,35 & 22,85 \\
\hline Panicum elephantipes Trin. & Poaceae & 549,42 & 8,81 & 40,06 & 6,42 & 15,23 \\
\hline Hymenachne donacifolia (Raddi) Chase & Poaceae & 193 & 3,10 & 42,02 & 6,74 & 9,84 \\
\hline Cissus spinosa Cambess. & Vitaceae & 214,79 & 3,45 & 39,2 & 6,26 & 9,71 \\
\hline Aspilia latissima Malme & Asteraceae & 274,37 & 4,40 & 29,6 & 4,65 & 9,06 \\
\hline Hydrocotyle ranunculoides L.f. & Araliaceae & 337,79 & 5,42 & 17,06 & 2,73 & 8,15 \\
\hline Polygonum ferrugineum Wedd. & Polygonaceae & 202,44 & 3,25 & 22,01 & 3,53 & 6,78 \\
\hline Ipomoea cordatotriloba Dennst. & Convolvulaceae & 157,06 & 2,52 & 25,06 & 4,01 & 6,53 \\
\hline Hymanachne pernanbucensis (Spreng.) Zuloaga & Poaceae & 109,37 & 1,75 & 26,06 & 4,17 & 5,93 \\
\hline Cayaponia podantha Cogn. & Cucurbitaceae & 70,22 & 1,13 & 27,03 & 4,33 & 5,46 \\
\hline Panicum dichotomiflorum Michx. & Poaceae & 56,03 & 0,90 & 22,1 & 3,53 & 4,43 \\
\hline Mikania micrantha Kunth & Asteraceae & 92,79 & 1,49 & 18,03 & 2,89 & 4,38 \\
\hline Hibiscus sp. & Malvaceae & 60,73 & 0,97 & 21,01 & 3,37 & 4,34 \\
\hline Stephostachys mertensii (Roth) Zuloaga \& Morrone & Poaceae & 21,89 & 0,35 & 23,01 & 3,69 & 4,04 \\
\hline Polygonum hispidum Kunth & Poaceae & 32,38 & 0,52 & 18,03 & 2,89 & 3,41 \\
\hline Passiflora misera Kunth & Passifloraceae & 9,09 & 0,15 & 19,1 & 3,05 & 3,20 \\
\hline Cyclanthera hystrix (Gill.) Arn. & Cucurbitaceae & 6 & 0,10 & 18,01 & 2,89 & 2,99 \\
\hline Pavonia laetevirens R.E.Fr. & Malvaceae & 69,27 & 1,11 & 9,97 & 1,61 & 2,72 \\
\hline Ludwigia helminthorrhiza (Mart.) H.Hara & Onagraceae & 121,52 & 1,95 & 4,2 & 0,64 & 2,59 \\
\hline Crateva tapia L. & Capparaceae & 39,82 & 0,64 & 6,09 & 0,96 & 1,60 \\
\hline Ipomoea carnea subsp. fistulosa (Mart. ex Choisy) D.F.Austin & Convolvulaceae & 35,12 & 0,56 & 5,2 & 0,80 & 1,37 \\
\hline Oxycaryum cubense (Poepp. \& Kunth) Lye & Cyperaceae & 30,17 & 0,48 & 5,2 & 0,80 & 1,29 \\
\hline Wolffiella lingulata (Hegelm.) Hegelm. & Araceae & 1,24 & 0,02 & 7,2 & 1,12 & 1,14 \\
\hline Salvinia minima Baker & Salviniaceae & 12,47 & 0,20 & 4,2 & 0,64 & 0,84 \\
\hline Byttneria filipes Mart. ex K.Schum. & Malvaceae & 11,32 & 0,18 & 4,1 & 0,64 & 0,82 \\
\hline Azolla filiculoides Lam. & Salviniaceae & 7,98 & 0,13 & 3,2 & 0,48 & 0,61 \\
\hline Enydra radicans (Willd.) Lack & Asteraceae & 17,53 & 0,28 & 2,07 & 0,32 & 0,60 \\
\hline Pistia stratiotes L. & Araceae & 2,76 & 0,04 & 2,02 & 0,32 & 0,37 \\
\hline Lemna valdiviana Phil. & Araceae & 0,19 & 0,00 & 2,07 & 0,32 & 0,32 \\
\hline Rhabdadenia madida (Vell.) Miers & Apocynaceae & 5,44 & 0,09 & 1,01 & 0,16 & 0,25 \\
\hline \multirow[t]{2}{*}{ Phyllanthus fluitans Benth. ex Müll.Arg. } & Phyllanthaceae & 0,57 & 0,01 & 1,01 & 0,16 & 0,17 \\
\hline & & 6234,64 & 100 & 626,25 & 100 & 200 \\
\hline
\end{tabular}


Tabela 2: Parâmetros fitossociológicos (Coberturas absoluta (CA) e relativa (CR), freqüências absoluta (FA) e relativa (FR) e valor de importância (VI)) obtidos através do método das parcelas para a comunidade de macrófitas aquáticas amostrada às margens do rio Miranda, Pantanal, MS, Brasil.

\begin{tabular}{|c|c|c|c|c|c|c|}
\hline Espécie & Família & CA & CR & FA & $\mathbf{F R}$ & VI \\
\hline Polygonum acuminatum Kunth & Polygonaceae & 1444,7 & 23,17 & 64,2 & 10,27 & 33,45 \\
\hline Commelina schomburgkiana Klotzsch & Commelinaceae & 1143,02 & 18,33 & 45,02 & 7,22 & 25,56 \\
\hline Merremia umbellata (L.) Hallier f. & Convolvulaceae & 904,15 & 14,50 & 52,1 & 8,35 & 22,85 \\
\hline Panicum elephantipes Trin. & Poaceae & 549,42 & 8,81 & 40,06 & 6,42 & 15,23 \\
\hline Hymenachne donacifolia (Raddi) Chase & Poaceae & 193 & 3,10 & 42,02 & 6,74 & 9,84 \\
\hline Cissus spinosa Cambess. & Vitaceae & 214,79 & 3,45 & 39,2 & 6,26 & 9,71 \\
\hline Aspilia latissima Malme & Asteraceae & 274,37 & 4,40 & 29,6 & 4,65 & 9,06 \\
\hline Hydrocotyle ranunculoides L.f. & Araliaceae & 337,79 & 5,42 & 17,06 & 2,73 & 8,15 \\
\hline Polygonum ferrugineum Wedd. & Polygonaceae & 202,44 & 3,25 & 22,01 & 3,53 & 6,78 \\
\hline Ipomoea cordatotriloba Dennst. & Convolvulaceae & 157,06 & 2,52 & 25,06 & 4,01 & 6,53 \\
\hline Hymanachne pernanbucensis (Spreng.) Zuloaga & Poaceae & 109,37 & 1,75 & 26,06 & 4,17 & 5,93 \\
\hline Cayaponia podantha Cogn. & Cucurbitaceae & 70,22 & 1,13 & 27,03 & 4,33 & 5,46 \\
\hline Panicum dichotomiflorum Michx. & Poaceae & 56,03 & 0,90 & 22,1 & 3,53 & 4,43 \\
\hline Mikania micrantha Kunth & Asteraceae & 92,79 & 1,49 & 18,03 & 2,89 & 4,38 \\
\hline Hibiscus sp. & Malvaceae & 60,73 & 0,97 & 21,01 & 3,37 & 4,34 \\
\hline Stephostachys mertensii (Roth) Zuloaga \& Morrone & Poaceae & 21,89 & 0,35 & 23,01 & 3,69 & 4,04 \\
\hline Polygonum hispidum Kunth & Poaceae & 32,38 & 0,52 & 18,03 & 2,89 & 3,41 \\
\hline Passiflora misera Kunth & Passifloraceae & 9,09 & 0,15 & 19,1 & 3,05 & 3,20 \\
\hline Cyclanthera hystrix (Gill.) Arn. & Cucurbitaceae & 6 & 0,10 & 18,01 & 2,89 & 2,99 \\
\hline Pavonia laetevirens R.E.Fr. & Malvaceae & 69,27 & 1,11 & 9,97 & 1,61 & 2,72 \\
\hline Ludwigia helminthorrhiza (Mart.) H.Hara & Onagraceae & 121,52 & 1,95 & 4,2 & 0,64 & 2,59 \\
\hline Crateva tapia L. & Capparaceae & 39,82 & 0,64 & 6,09 & 0,96 & 1,60 \\
\hline Ipomoea carnea subsp. fistulosa (Mart. ex Choisy) D.F.Austin & Convolvulaceae & 35,12 & 0,56 & 5,2 & 0,80 & 1,37 \\
\hline Oxycaryum cubense (Poepp. \& Kunth) Lye & Cyperaceae & 30,17 & 0,48 & 5,2 & 0,80 & 1,29 \\
\hline Wolffiella lingulata (Hegelm.) Hegelm. & Araceae & 1,24 & 0,02 & 7,2 & 1,12 & 1,14 \\
\hline Salvinia minima Baker & Salviniaceae & 12,47 & 0,20 & 4,2 & 0,64 & 0,84 \\
\hline Byttneria filipes Mart. ex K.Schum. & Malvaceae & 11,32 & 0,18 & 4,1 & 0,64 & 0,82 \\
\hline Azolla filiculoides Lam. & Salviniaceae & 7,98 & 0,13 & 3,2 & 0,48 & 0,61 \\
\hline Enydra radicans (Willd.) Lack & Asteraceae & 17,53 & 0,28 & 2,07 & 0,32 & 0,60 \\
\hline Pistia stratiotes L. & Araceae & 2,76 & 0,04 & 2,02 & 0,32 & 0,37 \\
\hline Lemna valdiviana Phil. & Araceae & 0,19 & 0,00 & 2,07 & 0,32 & 0,32 \\
\hline Rhabdadenia madida (Vell.) Miers & Apocynaceae & 5,44 & 0,09 & 1,01 & 0,16 & 0,25 \\
\hline \multirow[t]{2}{*}{ Phyllanthus fluitans Benth. ex Müll.Arg. } & Phyllanthaceae & 0,57 & 0,01 & 1,01 & 0,16 & 0,17 \\
\hline & & 6234,64 & 100 & 626,25 & 100 & 200 \\
\hline
\end{tabular}

Polygonum acuminatum pode ser frequentemente encontrada crescendo sobre solos férteis argilosos ao longo dos rios na região do Pantanal. O fato de suas sementes serem dispersas pela água, assim como observado para a maioria das espécies pertencentes a este gênero, justifica a ampla ocorrência desta espécie na região pantaneira (Pott \& Pott,
2000). O acentuado potencial de reprodução vegetativa apresentado pela espécie, pode de certa forma justificar seus elevados valores de cobertura (CA) e frequência absolutas (FA), já que onde ocorre a mesma costuma formar grandes manchas, cobrindo consideráveis trechos ao longo das margens do Rio Miranda nas proximidades da área de estudo. Pivari 
et al. (2008) reportam a ocorrência da espécie associada a ilhas flutuantes (baceiros), que se caracterizam por apresentar solos orgânicos.

Além de Polygonum acuminatum, C. schomburgkiana, M. umbellata e P. elephantipes também apresentam elevado potencial para reproduzir-se vegetativamente, tratando-se de uma estratégia de regeneração, que confere a estas espécies maiores vantagens na competição por espaço.

Dentre as formas biológicas encontradas, emergente foi a mais comum entre as espécies ocorrentes na área (18 espécies), seguida por anfíbia e flutuante livre representadas respectivamente por oito e sete espécies (Figura 3). A inundação do rio Miranda tem amplitudes baixas, com cerca de 1 a $2 \mathrm{~m}$ acima dos níveis onde estão os ambientes pesquisados. A maioria das espécies emergentes encontradas é capaz de apresentar esse crescimento durante o período da cheia e manter sua dominância durante o máximo do pulso de inundação. Como muitas dessas espécies podem sobreviver durante o período da seca (e.g. Polygonum spp), o que confere alta competitividade a essa forma de vida nesse tipo de ambiente.

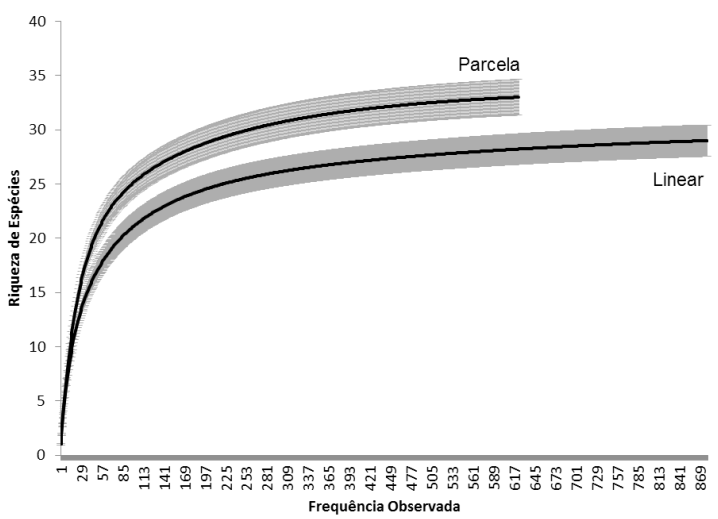

Figura 3: Curva de rarefação para ambas as metodologias utilizadas (Parcela e Interseção linear), indicando tendência à estabilização para a comunidade de macrófitas aquáticas amostrada às margens do rio Miranda, Pantanal, MS, Brasil.

Estudos realizados na região (Pott et al., 1992, 1999) e em outras partes do país (Costa et al., 2003) também listam emergente como a forma biológica mais freqüente. As espécies emergentes se caracterizam por estarem enraizadas no fundo, parcialmente submersas e parcialmente emersas.

O método da interseção linear revelou a ocorrência de 29 espécies, das quais Alter- nanthera aquatica Chodat e Lippia alba (Mill .) N.E. Br. ex Britton \& P. Wilson foram exclusivas nesta amostragem. Já para o método das parcelas, foram registradas 33 espécies, sendo Cyclanthera hystrix (Gillies) Arn., Ipomoea cordatotriloba Dennstedt, Passiflora misera Kunth, Pavonia laetevirens R. E. Fr., Pistia stratiotes L. e P. hispidum Kunth registradas apenas nesta metodologia. Os dados da riqueza em razão da suficiência amostral foram confirmados na curva de rarefação, que demonstra uma tendência à estabilização para ambos os métodos, indicando não haver necessidade de aumentar o número de unidades amostrais (Figura 4).

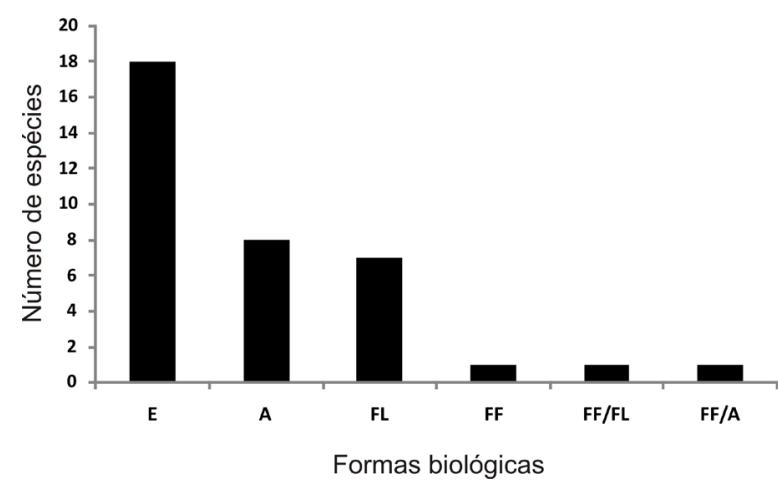

Figura 4: Formas biológicas observadas na comunidade de macrófitas aquáticas amostrada às margens do rio Miranda, Pantanal, MS, Brasil: Emergente (E), Anfíbia $(A)$, Flutuante-livre (FL), Flutuante-fixa (FF), Flutuante-fixa/flutuante-livre (FF/FL) e Flutuante-fixa/anfíbia.

Provavelmente o tamanho da área amostrada justifique o menor número de espécies observadas através do método de interseção linear, já que, através do método de parcelas a área amostrada é cerca de três vezes maior. Ainda assim, quando levado em consideração o índice de diversidade de Shannon, os valores obtidos mostram-se muito próximos em ambos os métodos utilizados (Tabela 3).

\begin{tabular}{ll}
\hline Método & $\begin{array}{l}\text { Diversidade de } \\
\text { Shannon }\left(\mathrm{H}^{\prime}\right)\end{array}$ \\
\hline Interseção linear & 2,44 \\
Parcelas & 2,47 \\
\hline
\end{tabular}

Tabela 3: Parâmetros fitossociológicos (Coberturas absoluta (CA) e relativa (CR), freqüências absoluta (FA) e relativa (FR) e valor de importância (VI)) obtidos através do método das parcelas para a comunidade de macrófitas aquáticas amostrada às margens do rio Miranda, Pantanal, MS, Brasil. 
Concluímos que tanto o método da interseção linear quanto o das parcelas são indicados para estudos quantitativos envolvendo macrófitas aquáticas, uma vez que os resultados obtidos no presente estudo foram similares.

Estudos quantitativos envolvendo macrófitas aquáticas tornam-se extremamente necessários para que possamos compreender melhor os padrões estruturais destas comunidades, ressaltando ainda que as áreas úmidas estão entre os ambientes que sofrem maior pressão devido à ação antrópica.

\section{ReferÊnCIAS}

Adrianarivo, J. 1993. Using GIS to valuate the crown-line intercept sampling method in orest survey. Forest Ecology and Management Amsterdam 58:87-103.

Bove, C. P.; Gil, A. S. B.; Moreira, C. B.; Anjos, R. F. B. 2003. Hidrófitas fanerogâmicas de ecossistemas aquáticos temporários da planície costeira do Estado do Rio de Janeiro, Brasil. Acta Botanica Brasilica 17: 119-135.

Costa, C. S. B.; Irgang, B.; Peixoto, A. R. \& Marangoni, J. C. 2003. Composição florística das formações vegetais sobre uma turfeira topotrófica da planície costeira do Rio Grande do Sul, Brasil. Acta Botanica Brasilica 17(2): 203-212.

Damasceno-Junior, G.A.; Pott, A. 2011. Métodos de Amostragem em estudos fitossociológicos sugeridos para o Pantanal. In: Felfili, J.M.; Eisenlohr, P.V.; Melo, M.M.R.F.; Andrade, L.A.; Meira-Neto, J.A.A.(Org.). Fitossociologia no Brasil: métodos e estudos de casos. Viçosa: Editora UFV: 295-323.

Esteves, F. A. 1998. Fundamentos de Limnologia. Rio de Janeiro: Editora Interciência, 602p.

Frey, R. 1995. Flora and vegetation of "Las Piedritas" and the margin of Laguna Cáceres, Puerto Suárez, Bolivian Pantanal. Bulletin Torrey Botanical Club 122:314-319.

Hammer, Ø., Harper, D.A.T., and P. D. Ryan, 2001. PAST: Paleontological Statistics Software Package for Education and Data Analysis. Palaeontologia Electronica 4(1): 9pp. http://palaeo-electronica. org/2001_1/past/issue1_01.htm
Hamilton, S. K.; Sippel, S. J.; Melak, J. M. 1996. Inundation patterns in the Pantanal wetland of South America determined from passive microwave remote sensing. Archives of Hidrobiology 37: (1) 1-23).

Irgang, B. E., Pedralli, G., Waechter, J. I. 1984. Macrófitos aquáticos da Estação Ecológica do Taim, Rio Grande do Sul, Brasil. Rossléria, 6(1): 395-405.

Junk, W. J.; Piedade, M. T. F. 1993. Biomass and primary-production of herbaceous plant communities in the Amazon floodplain. Hydrobiologia, 263: 155-162.

Köppen, W. 1948. Climatologia com um estúdio de los climas de la tierra. Ed. Fondo Cultura Econômica, Ciudad de México.

Meirelles, M. L.; Oliveira, R. C.; Ribeiro, J. F.; Vivaldi, L. J.; Rodrigues, L.A.; Silva, G. P. 2002. Utilização do método de interseção na linha em levantamento quantitativo do estrato herbáceo do cerrado. Boletim Herbário Ezechias Paulo Heringer 9:60-68.

Meirelles, M. L.; Guimarães A. J. M.; Oliveira, R. C.; Araújo, G. M.; Ribeiro, J. F. 2004. Impactos sobre o estrato herbáceo de áreas úmidas do Cerrado. In: Aguiar, L. M. S. Camargo, A. J. A. (Org.). Cerrado: ecologia e caracterização. 1 ed. Planaltina: Embrapa Cerrados 1: 41-68.

Mueller-Dombois, D.; Ellenberg. H. 1974. Aims and methods of vegetation ecology. New York: Wiley. 547 p.

Munhoz, C. B.; Felfili, J. M. 2004. Composição florística do estrato herbáceo-subarbustivo em uma área de campo sujo na fazenda água limpa no Distrito Federal, Brasil. Boletim Herbário Ezechias Paulo Heringer 13:61-84.

Munhoz, C. B. 2006a. Fitossociologia do estrato herbáceo-subarbustivo de uma área de campo sujo no Distrito Federal, Brasil. Acta Botanica Brasílica 20(3):671-685.

Munhoz, C. B. 2006b. Floristics of the herbaceous and subshrub layer of a moist grassland in the Cerrado Biosphere reserve (Alto Paraíso de Goiás), Brazil. Edinburgh Journal of Botany 63: 343-354

Munhoz, C. B. 2007. Florística do estrato herbáceo - subarbustivo de um campo limpo úmido em Brasília, Brasil. Biota Neotropica 7(3): 205 - 215. 
Munhoz, C. B. 2008. Fitossociologia do estrato herbaceo-subarbustivo em campo limpo úmido no Brasil Central. Acta Botanica Brasilica, v. 22, p. 905-913, 2008.

Nemati, N.; Goetz, H. 1995. Relationships of overstory to understory cover variables in a Ponderosa pine/Gambel oak ecosystem. Plant Ecology 119(1): 15-21.

Pedrali, G. 1992. Macrófitos aquáticos: centros de diversidade. Ciência Hoje 14:(79) 56-57.

Pivari, M. O.; Pott, V. J.; Pott, A. 2008. Macrófitas Aquáticas de Ilhas Flutuantes (Baceiros) nas Sub-regiões do Abobral e Miranda, Pantanal, MS, Brasil.. Acta Botanica Brasilica 22: 563-571.

Pott, V. J.; Bueno, N. C.; Pereira, R. A. C.; Salis, S. M.; Vieira, N. L. 1989. Distribuição de macrófitas aquáticas numa lagoa na fazenda Nhumirim, Nhecolândia, Pantanal, MS. Acta Botanica Brasilica 3(2) Supl.: 153-168.

Pott, V. J.; Bueno, N. C.; Silva, M. P. da. 1992. Levantamento florístico e fitossociológico de macrófitas aquáticas em lagoas da Fazenda Leque, Pantanal, Mato Grosso do Sul. Anais $8^{\circ}$ Congresso da Sociedade Botânica de São Paulo (SBSP): 91-99.

Pott, V. J.; Cervi, A. C.; Bueno, N. C.; Pott, A. 1999. Dinâmica da vegetação aquática de uma lagoa permanente na fazenda Nhumirim, Pantanal da Nhecolândia - MS. In: Simpósio Sobre Recursos Naturais e Sócio Econômicos do Pantanal. Corumbá, Embrapa Pantanal 227-235.

Pott, V. J.; Pott, A. 2000. Plantas Aquáticas do Pantanal. Brasília: Embrapa, 404p.
Prado, D. E.; Gibbs, P. E.; Pott, A.; Pott, V. J. 1992. The Chaco-Pantanal transition in southern Mato Grosso, Brazil. In: Furley P. A.; Ratter, J. A. (eds.), Nature and dynamics of forest-savanna boundaries. Chapman \& Hall, London.

Salis, S. M. 2000. Fitossociologia da vegetação arbórea no entorno de uma lagoa no Pantanal Mato-grossense, Brasil. Naturalia 25:225-241.

Schessl, M. 1999. Floristic composition and structure os floodplain vegetation in the northern Pantanal of Mato Grosso, Brazil. Phyton 39(2):303-336.

Schulz, M.; Kozerski, H.P.; Pluntke, T.; Rinke, K. 2003. The influence of macrophytes on sedimentation and nutrient retention in the lower river Spree (Germany). Water Res., v.37, p.569-578.

Soriano, B. M. A. 1999. Caracterização climática da sub-região da Necolândia, Pantanal, MS. In Anais do II Simpósio sobre recursos naturais e sócio-econômicos do Pantanal (M. Dantas, J. B. Catto \& E. K. Resende, coords.) Embrapa Pantanal, Corumbá, p:151-158.

Tansley, A. G.; Chipp, T. F. 1926. Aims and methods in study of vegetation. London: British Empire Veg Chee and Crown Agents for the Colonies. 383p.

Thomaz, S.M \& Bini, L.M. 2003. Análise crítica dos estudos sobre macrófitas aquáticas desenvovidos no Brasil. In: S.M. Thomaz \& L.M. Bini (eds.). Ecologia e manejo de macrófitas aquáticas. Editora da Universidade Estadual de Maringá, Maringá. Pp. 19-38.

Wolf, J. D. 1998. Species composition and structure of woody vegetation of the Middle Casamance region (Senegal). Forest Ecology and Management, Amsterdam 111:249-264. 